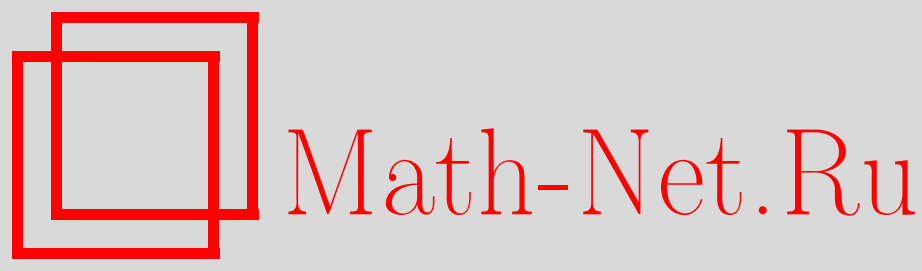

С. Б. Лебле, Квантовые поправки к статическим решениям моделей синус-Гордон и Нама в терминах обобщенной дзета-функции, ТМФ, 2009, том 160, номер 1, 122132

DOI: https://doi.org/10.4213/tmf6384

Использование Общероссийского математического портала Math-Net.Ru подразумевает, что вы прочитали и согласны с пользовательским соглашением http://www.mathnet.ru/rus/agreement

Параметры загрузки:

IP: 52.6 .47 .48

26 апреля 2023 г., 18:23:48

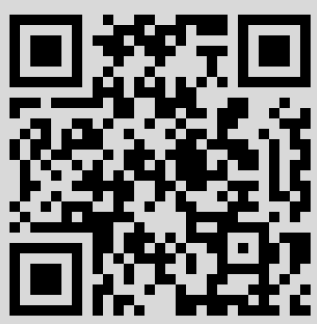




\title{
КВАНТОВЫЕ ПОПРАВКИ К СТАТИЧЕСКИМ РЕШЕНИЯМ МОДЕЛЕЙ СИНУС-ГОРДОН И НАМА В ТЕРМИНАХ ОБОБЩЕННОЙ ДЗЕТА-ФУНКЦИИ
}

\begin{abstract}
В терминах класса нелинейных уравнений Клейна-Гордона-Фока рассмотрены одномерные модели Янга-Миллса-Нама и синус-Гордон. Квазиклассическое квантование модели выполнено с помощью метода обобщенной дзета-функции, а представления этой квантовой теории построены с помощью диагональной функции Грина для уравнения теплопроводности с эллиптическим потенциалом с применением решений уравнения Эрмита. Сформулирован альтернативный подход, основанный на функции Бейкера-Ахиезера для уравнения Кадомцева-Петвиашвили. Вычислены квантовые поправки к действию моделей Нама и синус-Гордон. Исследованы поля из класса эллиптических функций. В связи с возможными применениями квантованных солитонов модели синус-Гордон в физике твердого тела с помощью модели Френкеля-Конторовой или с помощью других моделей произведен учет дополнительных переменных произвольных размерностей. Для модели Нама, поле которой по построению представлено эллиптическим интегралом (лемнискатой), масса поля Янга-Миллса совпадает с поправкой, вычисляемой с помощью гиперэллиптического интеграла.
\end{abstract}

Ключевые слова: квантовые поправки, уравнение синус-Гордон, модель Нама, обобщенная дзета-функция, эллиптические решения, уравнение Эрмита.

\section{1. ВВЕДЕНИЕ}

1.1. Общие замечания. В настоящей работе рассматривается одномерная полевая теория, которая базируется на нелинейных уравнениях Клейна-Гордона-Фока и появляется, например, в моделях Френкеля-Конторовой для дислокационных дефектов кристаллических структур [1] или в релятивистских моделях [2], принимая вид уравнения синус-Гордон (СГ). Также учитываются дополнительные размерности, с помощью которых удается проследить за физическим содержанием соответствующих явлений. Основная задача настоящей работы состоит в вычислении квантовых поправок к действию с помощью дзета-функционального представления однопетлевой поправки в фейнмановской теории в формализме ядра уравнения теплопроводности. Выводятся явные выражения, которые могут быть использованы

${ }^{*}$ Gdansk University of Technology, Gdansk, Poland. E-mail: leble@mif.pg.gda.pl 
впоследствии в теории твердого тела в различных размерностях, в которой делается различие между вкладами от кинков и от эллиптических солитонов.

Теория поля Янга-Миллса обладает помимо всего прочего редукциями к одномерным моделям [3]. Также возможно вложить эту теорию в объемлющую многомерную теорию: конструкция Атьи-Дринфельда-Хитчина-Манина-Нама при этом может возникать в качестве условия эквивалентности между двумя наборами уравнений самодуальности: в размерности 1, как описано выше, и в размерности 3 [4]. Одномерная модель представляет собой частный случай $(m=0)$ другой хорошо известной модели, а именно модели Гинзбурга-Ландау (ГЛ), также известной как $\phi^{4}$-модель, широко применяемой в теории фазовых переходов. Отсюда вытекает, что масса поля Янга-Миллса в квазиклассическом приближении совпадает с квантовой поправкой к действию модели Нама.

Отметим, что наш выбор вакуумного состояния, который отвечает фиксации сдвига потенциала на константу в соответствующем вакуумном операторе $D_{0}(5)$, позволяет избежать дополнительной перенормировки.

Класс нелинейных уравнений в случае статических одномерных решений сводится к уравнениям

$$
\phi^{\prime \prime}-V^{\prime}(\phi)=0, \quad \phi=\phi(x), \quad x \in \mathbb{R} .
$$

Первый интеграл уравнения (1), $W=\phi^{2} / 2-V(\phi)$, задает дифференциальное уравнение с хорошо известным семейством решений: постоянные, кинки, периодические и так называемые "уходящие" решения [5]. Он содержит постоянную $W$, которая в случае моделей Нама и СГ выбирается в соответствии с моделью ГЛ:

$$
V_{\mathrm{N}}(\phi)=\frac{\phi^{4}}{2}-W, \quad W=-\frac{b^{4}}{2}, \quad V_{\mathrm{SG}}(\phi)=\frac{2 m^{4}}{3 g}\left(1+\cos \left(\frac{1}{m} \sqrt{\frac{3 g}{2}} x\right)\right)
$$

Метод, предлагаемый в настоящей работе, открывает новые возможности: например, с его помощью можно вычислять квантовые поправки к периодическим решениям и к матричным моделям сходной структуры. Такие задачи были поставлены в обзорной статье [5]. Отметим, что решение модели Нама с необходимостью выражается через эллиптические функции (см. результаты работ [6], [7]). Необходимость квантования именно эллиптических решений, называемых “твистованными кинками", объяснена в работе [6].

\section{2. Фейнмановское квантование и обобщенная дзета-функция Рима-} на. Интерес к фейнмановскому формализму квантования для классического поля снова появился в последнее время в связи с исследованием квазиклассического условия квантования в суперсимметричных теориях [8]. Теория квантовых поправок в этом формализме была инициирована работой [9]. В работах [10] с помощью дзета-функции Римана были вычислены поправки к нескольким классическим решениям в размерности $d>1$. Наиболее известными из этих поправок являются поправки к кинку - сепаратрисному решению модели ГЛ [11]. 
Приближенные квантовые поправки к решениям уравнения (1) получаются с помощью фейнмановского функционального интеграла, вычисляемого методом стационарной фазы. В результате получается следующее соотношение:

$$
e^{-S_{\mathrm{qu}} / \hbar} \simeq \frac{A}{\sqrt{\operatorname{det} D}},
$$

где $S_{\text {qu }}$ - квантовое действие, соответствующее потенциалу $V(\phi), A$ - некоторая величина, определяемая вакуумным состоянием при $V(\phi)=0, \operatorname{det} D-\operatorname{oпределитель~}$ оператора

$$
D=-\partial_{x}^{2}-\Delta_{y}+V^{\prime \prime}(\phi(x)) .
$$

Эта поправка возникает при учете второй вариационной производной от функционала квантового действия. Аргумент $y \in \mathbb{R}^{d-1}$ обозначает поперечные переменные, от которых решение $\phi(x)$ не зависит. Соотношение вида (3) справедливо для кван-

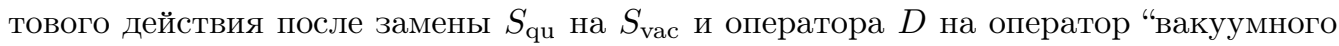
состояния" $D_{0}=-\partial_{x}^{2}-\Delta_{y}$. Тогда квантовая поправка $\Delta S_{\mathrm{qu}}=S_{\mathrm{qu}}-S_{\mathrm{vac}}$ получается двукратным применением формулы (3) и имеет вид

$$
\Delta S_{\mathrm{qu}}=\frac{\hbar}{2} \ln \frac{\operatorname{det} D}{\operatorname{det} D_{0}} .
$$

Обобщенная дзета-функция появляется в задачах квантовой теории, основанных на фейнмановском функциональном интеграле в квазиклассическом приближении. Пусть множество $\left\{\lambda_{n}\right\}=S$ представляет собой спектр линейного оператора $L$, тогда с помощью формального суммирования имеем $\sum_{\lambda_{n} \in S} \ln \lambda_{n}=\ln (\operatorname{det} L)$.

Обобщенная дзета-функция Римана $\zeta_{L}(s)$ формально задается равенством

$$
\zeta_{L}(s)=\sum_{\lambda_{n} \in S} \lambda_{n}^{-s} .
$$

Это определение можно интерпретировать как аналитическое продолжение в комплексную плоскость по переменной $s$ из полуплоскости $\operatorname{Re} s>\sigma$, в которой ряд в выражении (6) сходится. Дифференцируя соотношение (6) по $s$ в точке $s=0$, получаем

$$
\ln (\operatorname{det} L)=\zeta_{L}^{\prime}(0)
$$

Обобщенная дзета-функция (6) допускает представление с помощью диагонали функции Грина $g_{L}$ оператора $\partial_{t}+L$. Связь с диагональю функции Грина (формализм ядра уравнения теплопроводности) применялась в квантовой теории, начиная с работ Фока [12], с помощью спектрального разложения по нормированным собственным функциям $\psi_{n}(\mathbf{r})$ :

$$
g_{L}\left(t, \mathbf{r}, \mathbf{r}_{0}\right)=\sum_{n} e^{-\lambda_{n} \mathbf{t}} \psi_{n}(\mathbf{r}) \psi_{n}^{*}\left(\mathbf{r}_{0}\right)
$$

Возвращаясь к операторам $D, D_{0}(4)$ поставим теперь основную задачу для одномерного оператора

$$
D_{1}=-\frac{d^{2}}{d x^{2}}+u(x)
$$


с периодическим потенциалом $u(x)=V^{\prime \prime}(\phi(x))$ и определим

$$
\gamma_{L}(t)=\int g_{L}(t, x, x) d x=\sum_{k} e^{-\lambda_{k} t} .
$$

Из соотношения для функции $\gamma_{D}(t)$ следует важное свойство мультипликативности.

Если оператор $D$ представляется в виде суммы двух дифференциалъных операторов, $D_{1}$ и $D_{2}$, зависящих от разных переменных, имеет место равенство $\gamma_{D}(t)=\gamma_{D_{1}}(t) \gamma_{D_{2}}(t)$.

Для применяемого в дальнейшем в качестве вакуумного $d$-мерного оператора $D_{\nu}$ с постоянным потенциалом $\nu$ свойство мультипликативности дает

$$
\gamma_{D_{\nu}}(t)=4 \pi^{(1-d) / 2} \frac{\Gamma(s+(1-d) / 2)}{\Gamma(s)} \nu^{(d-1-2 s) / 2} .
$$

Наконец с помощью преобразования Меллина получаем обобщенную дзета-функцию

$$
\zeta(s)=\frac{1}{\Gamma(s)} \int_{0}^{+\infty} t^{s-1}\left(\gamma_{D}(t)-\gamma_{D_{0}}(t)\right) d t,
$$

соответствующую (5).

\section{2. СТАТИЧЕСКИЕ РЕШЕНИЯ МОДЕЛЕЙ НАМА И СГ}

2.1. Случай модели Нама. Рассмотрим полную систему уравнений ЯнгаМиллса в евклидовом пространстве размерности 4

$$
D_{\mu} T_{\mu \nu}=\partial_{\mu} T_{\mu \nu}-i\left[T_{\mu}, T_{\mu \nu}\right]=0
$$

для калибровочных полей $T_{\mu}=T_{\mu}^{+}$и $T_{\mu \nu}=T_{\nu, \mu}-T_{\mu, \nu}-i\left[T_{\mu}, T_{\nu}\right]$. Налагая условия, что все поля не зависят от переменных $x_{k}, k=1,2,3$, и полагая $x_{4}=z$, найдем, что уравнения самодуальности

$$
\frac{d T_{i}}{d z}= \pm \varepsilon_{i j k} T_{j} T_{k}
$$

отвечающие такой модели, приводят к уравнениям (13). В случае простейшего решения системы (14) $T_{i}=\phi_{i} \sigma_{i}$, построенного с помощью матриц Паули, получаем систему уравнений Эйлера для $\phi_{i}(y)$, решения которой выражаются через функции Якоби [13].

Для уравнений второго порядка аналогичный анзац

$$
T_{i}=\phi(z) \alpha_{i}, \quad i=1,2,3,
$$

с постоянными матрицами $\alpha_{i}$ и подходящим выбором масштабных преобразований приводит к уравнению

$$
\phi^{\prime \prime}(z)=2 \phi^{3} .
$$

Уравнение (16) оказывается представителем класса нелинейных уравнений (1) с $V^{\prime}(\phi)=2 \phi^{3}$. Решение (16) оказывается специальным случаем статической модели СГ с $m=0, g=2$ (см. (2)). 
Решение уравнения (16) выражается через эллиптические функции [14], а именно функции, обратные эллиптическому интегралу (лемнискате),

$$
\int_{0}^{\phi} \frac{d \phi}{\sqrt{\phi^{4}-b^{4}}}=\frac{1}{b} \int_{0}^{\phi / b} \frac{d t}{\sqrt{\left(t^{2}-1\right)\left(t^{2}+1\right)}}=z,
$$

и приводится к эллиптическому синусу sn с чисто мнимым модулем $k=i$,

$$
\phi=b \operatorname{sn}(i b z ; i) \text {. }
$$

Этой функции соответствует функция Вейерштрасса с инвариантами $g_{2}=b^{4}, g_{3}=0$, что непосредственно приводит к решению в виде, приведенном в работе [4]. В случае модели Нама с помощью квантовых поправок удается вычислить массу поля Янга-Миллса в квазиклассическом представлении.

Применим выражение для потенциала в терминах решения (18):

$$
V^{\prime \prime}\left(\phi_{0}(x)\right)=\left(\frac{\phi^{4}}{2}\right)^{\prime \prime}=6 \phi^{2}=-6 b^{2}\left(1-\operatorname{cn}^{2}(b z, i)\right) .
$$

2.2. Случай модели СГ. Кратко опишем модель СГ. Уравнение (1) интегрируется с потенциалом $V_{\mathrm{SG}}$ из (2), что дает в итоге дифференциальное уравнение с параметром $W$. Решение (1) задает периодическую функцию, выражающуюся через эллиптические функции Якоби. Для ее нахождения подставляем $\varphi=$ $\pm 2 m \sqrt{2 /(3 g)} \arcsin z$ и получаем

$$
\left(z^{\prime}\right)^{2}=m^{2}\left(1+\frac{3 W g}{4 m^{4}}-z^{2}\right)\left(1-z^{2}\right) .
$$

Решение уравнения (20) похоже на решение (18) и задается выражением

$$
z=k \operatorname{sn}(m x ; k), \quad k=\sqrt{1+\frac{3 g W}{4 m^{4}}},
$$

в котором $k$ - модуль эллиптической функции. Окончательно имеем

$$
\varphi= \pm 2 m \sqrt{\frac{2}{3 g}} \arcsin k \operatorname{sn}(m x ; k) .
$$

В случае $W=0, k=1$ нетривиальные решения интерпретируются как кинк и антикинк.

\section{3. ВЫРАЖЕНИЕ ДЛЯ ОБОБЩЕННОЙ ДЗЕТА-ФУНКЦИИ ЧЕРЕЗ УРАВНЕНИЕ ЭРМИТА}

3.1. Уравнение для диагонали функции Грина. Преобразование Лапласа для уравнения теплопроводности

$$
\left(\frac{\partial}{\partial t}-\frac{d^{2}}{d x^{2}}+u(x)\right) g\left(t, x, x_{0}\right)=\delta(t) \delta\left(x-x_{0}\right)
$$


приводит к уравнению для функции Грина $g\left(p, x, x_{0}\right)$ для спектральной задачи оператора $D_{1}(9)$.

В духе подхода Эрмита [15] преобразование Лапласа

$$
\hat{g}(p, x, x)=\int e^{-p t} g(t, x, x) d t=G(p, x)
$$

от диагонали функции Грина представляет собой решение билинейного уравнения

$$
2 G G^{\prime \prime}-\left(G^{\prime}\right)^{2}-4(u(x)+p) G^{2}+1=0, \quad G^{\prime}=\frac{d G(p, x)}{d x},
$$

которое может быть более эффективно решено в случаях безотражательных и конечнозонных потенциалов. Все необходимые классы решений модели можно запараметризовать с помощью универсальных представлений в терминах многочленов $P, Q$ :

$$
G(p, x)=\frac{P(p, z)}{2 \sqrt{Q(p)}},
$$

где $z=\mathrm{cn}^{2}(b x ; k)$ в периодическом случае, или

$$
b^{2}\left(\rho(z)\left(2 P P^{\prime \prime}-\left(P^{\prime}\right)^{2}\right)+\rho^{\prime}(z) P P^{\prime}\right)-(p+u(z)) P^{2}+Q=0,
$$

штрихи обозначают производные по $z, \rho(z)=z(1-z)\left(1-k^{2}+k^{2} z\right)$,

$$
u(z)= \begin{cases}-6 b^{2}(1-z) & (\text { модель Нама) } \\ b^{2}\left(2 k^{2}-1-2 k^{2} z\right) & (\text { модель СГ). }\end{cases}
$$

Подставляя (29) в (26), получаем для коэффициентов при каждом степенном множителе $p=0,1,2$

$$
\begin{aligned}
-2 P_{1}(z)-u(z)+q_{2} & =0, \\
b^{2}\left(2 \rho(z) P_{1}^{\prime \prime}+\rho^{\prime}(z) P_{1}^{\prime}\right)-P_{1}^{2}-2 u(z) P_{1}+q_{1} & =0, \\
b^{2}\left(\rho(z)\left(2 P_{1} P_{1}^{\prime \prime}-P_{1}^{\prime 2}\right)+\rho^{\prime}(z) P_{1} P_{1}^{\prime}\right)-u(z) P_{1}^{2}+q_{0} & =0 .
\end{aligned}
$$

3.2. Случай модели СГ. Рассмотрим модель СГ. Подстановка

$$
P=p+P_{1}(z), \quad Q=p^{3}+q_{2} p^{2}+q_{1} p+q_{0}
$$

в (26) и (27) приводит к формулам

$$
P_{1}=k^{2} b^{2} z, \quad q_{2}=b^{2}\left(2 k^{2}-1\right), \quad q_{1}=b^{4} k^{2}\left(k^{2}-1\right), \quad q_{0}=0 .
$$

Наконец, интегрируя по периоду, можно выразить преобразование Лапласа $\widehat{\gamma}(p)$ через полные эллиптические интегралы $K(k), E(k)$ :

$$
\widehat{\gamma}(p)=\frac{2 K(k) p+b^{2}\left(E(k)-K(k)+2 k^{2} K(k)\right)}{2 \sqrt{p\left(p+b^{2} k^{2}\right)\left(p+b^{2} k^{2}-b^{2}\right)}} .
$$


Для двухзонного решения в общем случае имеем

$$
P=p^{2}+P_{1}(z) p+P_{2}(z), \quad Q=p^{5}+q_{4} p^{4}+q_{3} p^{3}+q_{2} p^{2}+q_{1} p+q_{0} .
$$

Подстановка выражений (32) в уравнения Эрмита приводит к системе уравнений

$$
\begin{array}{r}
-2 P_{1}-u+q_{4}=0, \\
-2 P_{2}-P_{1}^{2}-2 u P_{1}+b^{2}\left(2 \rho P_{1}^{\prime \prime}+\rho^{\prime} P_{1}^{\prime}\right)+q_{3}=0, \\
b^{2}\left(\rho\left(2 P_{2}^{\prime \prime}+2 P_{1} P_{1}^{\prime \prime}-\left(P_{1}^{\prime}\right)^{2}\right)+\rho^{\prime}\left(P_{2}^{\prime}+P_{1} P_{1}^{\prime}\right)\right)-2 P_{1} P_{2}-u\left(2 P_{2}+P_{1}^{2}\right)+q_{2}=0, \\
b^{2}\left(2 \rho\left(2 P_{1}^{\prime \prime} P_{2}-P_{1}^{\prime} P_{2}^{\prime}+P_{1} P_{2}^{\prime \prime}\right)+\rho^{\prime}\left(P_{1} P_{2}^{\prime}+P_{1}^{\prime} P_{2}\right)\right)-P_{2}^{2}-2 u P_{1} P_{2}+q_{1}=0, \\
b^{2}\left(\rho\left(2 P_{2} P_{2}^{\prime \prime}-P_{2}^{\prime 2}\right)+\rho^{\prime} P_{2} P_{2}^{\prime}\right)-u P_{2}^{2}+q_{0}=0,
\end{array}
$$

где мы опустили аргументы.

3.3. Случай модели Нама. В случае уравнения Нама имеем $q_{4}=0, q_{3}=$ $-21 b^{4}, q_{2}=0, q_{1}=108 b^{8}, q_{0}=0$, отсюда

$$
P_{1}(z)=-3 b^{2}(z-1), \quad P_{2}=18 b^{4} z^{2}-36 b^{4} z .
$$

Далее

$$
Q(p)=\prod_{i=1}^{5}\left(p-p_{i}\right)=p\left(p+3 b^{2}\right)\left(-p+3 b^{2}\right)\left(12 b^{4}-p^{2}\right),
$$

где простые корни $p_{i}$ многочлена $Q$ можно легко упорядочить для вещественных $b$.

Найдем выражение, определяющее $\widehat{\gamma}(p)$ с помощью интегрирования по периоду:

$$
\widehat{\gamma}(p)=\int \frac{\left(p^{2}-3 b^{2}(z-1) p+18 b^{4} z^{2}-36 b^{4} z\right) d x}{2 \sqrt{Q}} .
$$

Возвращаясь к переменной $z$, имеем $d z=d\left(\operatorname{cn}^{2} x\right)=-2 \operatorname{cn} x \operatorname{sn} x \operatorname{dn} x d x$, и снова интегрируя, получаем

$$
\widehat{\gamma}(p)=\frac{6 b^{4} K(i)+2 p^{2} K(i)+36 b^{4}(K(i)-E(i))-3 b^{2} p(E(i)-3 K(i))}{\sqrt{Q}},
$$

что с помощью (12) позволяет выразить дзета-функцию через полные эллиптические интегралы (лемнискаты) $K(i)$ и $E(i)$ [14]. Например, при $k=i$ получаем

$$
k^{\prime}=\sqrt{1-k^{2}}=\sqrt{2}, \quad K(i)=\frac{2}{1+\sqrt{2}} K\left(\frac{1-\sqrt{2}}{1+\sqrt{2}}\right) .
$$

\section{4. ФУНКЦИИ ГРИНА В ТЕРМИНАХ ФУНКЦИИ БЕЙКЕРА-АХИЕЗЕРА}

Рассмотрим функцию Грина для уравнения теплопроводности, определенную в разделе 1. Предлагаемый здесь метод основан на технике конечнозонного интегрирования уравнения Кадомцева-Петвиашвили [16]. Как было отмечено в разделе 3, одномерное уравнение теплопроводности (23) с периодическим потенциалом $u(x)=V^{\prime \prime}(\phi(x))$ представляет собой исходную часть задачи. 
Проинтегрируем (23) по $x$ в пределах $\left[x_{0}-\epsilon, x_{0}+\epsilon\right]$ :

$$
\begin{aligned}
\lim _{\epsilon \rightarrow 0} & \int_{x_{0}-\epsilon}^{x_{0}+\epsilon}\left(\frac{\partial}{\partial t}-\frac{d^{2}}{d x^{2}}+u(x)\right) g\left(t, x, x_{0}\right) d x= \\
\quad= & \lim _{\epsilon \rightarrow 0}\left[-\frac{d g\left(t, x_{0}+\epsilon, x_{0}\right)}{d x}+\frac{d g\left(t, x_{0}-\epsilon, x_{0}\right)}{d x}\right]=\delta(t) .
\end{aligned}
$$

Предполагается, что функция $g\left(t, x, x_{0}\right)$ непрерывна при $t>0$.

Решения уравнения (23) с нулевой правой частью выражаются в терминах функций Бейкера-Ахиезера (БА $\psi_{s}(x, t ; P)$, построенных с помощью многочлена $q(s)$ от локального параметра $s$ на римановой поверхности. Общий формализм для этой конструкции хорошо известен (см., например, [16]), а список соответствующих формул можно найти в работе [7].

Функция БА (формула Итса-Матвеева) для случая модели СГ выражается в терминах тета-функций Якоби и задает решение соответствующего уравнения Ламе с $u_{L}(x)=2 k^{2}\left(1-\mathrm{cn}^{2}(x, k)\right)$ и спектральным параметром $h$ [14]:

$$
\psi_{+}(x ; h)=\frac{\exp \left[\eta\left(2 v \rho+\rho^{2}\right) /(2 \omega)+v \zeta\left(\wp^{-1}\left(3-2\left(1+k^{2}\right)\right)\right)\right] \vartheta((v+\rho) /(2 \omega))}{\vartheta(v /(2 \omega))},
$$

где $\rho=\wp^{-1}\left(3 h-2\left(1+k^{2}\right)\right), \eta=\zeta(\omega), \omega=\wp^{-1}\left(2-k^{2}\right), v=\left(x-i K^{\prime}\right) / \sqrt{3}, K^{\prime}\left(k^{\prime}\right)-$ полный эллиптический интеграл, $\wp-$ функция Вейерштрасса, $\zeta(\omega)$ - дзета-функция Вейерштрасса $\left(\zeta^{\prime}=-\wp\right)$ [14]. Потенциал $u_{L}(x)$ стандартного уравнения Ламе отличается от $u(x)$ на константу и сдвиг: $u_{L}=b^{2} u+b^{2}$ (cp. с $\left.(27)\right)$. В этом разделе положим для простоты $b=1$. Спектральные параметры, таким образом, связаны между собой: $h=p+1$.

Базисная тета-функция представления (38) определяется формулой

$$
\vartheta(w)=\vartheta(w \mid \tau)=\sum_{m=-\infty}^{\infty} e^{i \pi\left(m^{2} \tau+2 m w\right)}
$$

где $e^{i \pi \tau}=\eta$ и величина $\operatorname{Im} \tau$ должна быть положительной для сходимости ряда. Ряд сходится достаточно быстро, поэтому представление (38) удобно для численных расчетов интегралов в формализме дзета-функции (12).

Функцию Грина $g_{h}$ спектральной задачи Ламе можно построить как произведение двух независимых решений $\psi_{+}, \psi_{-}$спектрального уравнения с одним и тем же $h$ :

$$
g_{h}\left(x, x_{0}\right)=\frac{1}{\mathbb{W}} \begin{cases}\psi_{+}(x ; h) \psi_{-}\left(x_{0} ; h\right), & x<x_{0} \\ \psi_{-}(x ; h) \psi_{+}\left(x_{0} ; h\right), & x>x_{0}\end{cases}
$$

Множитель $\mathbb{W}$ (вронскиан) добавлен для нормировки функции (40) таким образом, чтобы она имела единичный скачок первой производной по переменной $x$ :

$$
\lim _{\epsilon \rightarrow 0}\left[\frac{d g_{h}\left(x_{0}+\epsilon, x_{0}\right)}{d x}-\frac{d g_{h}\left(x_{0}-\epsilon, x_{0}\right)}{d x}\right]=-1 .
$$

Независимое решение $\psi_{-}(x, t ; h)$ можно выбрать нечетным относительно отражения $x \rightarrow-x$, т.е. его можно выразить через функцию $\vartheta(w+1 / 2)$.

5 Теоретическая и математическая физика, т. 160, № 1, 2009 г. 
Граничное условие при $t=0$ можно учесть с помощью интеграла по спектральному параметру $h$ (см. также (8)) от произведения линейно независимых решений:

$$
g\left(t, x, x_{0}\right)=\int \frac{1}{\mathbb{W}}\left\{\begin{array}{ll}
\Psi_{+}(x, t ; h) \Psi_{-}\left(x_{0}, t ; h\right), & x<x_{0} \\
\Psi_{+}\left(x_{0}, t ; h\right) \Psi_{-}(x, t ; h), & x>x_{0}
\end{array}\right\} d h,
$$

где $\Psi_{+}(x, t ; h)=e^{-h t} \psi_{+}(x ; h)$.

Проинтегрируем диагональные значения функции Грина (42) по периоду решения, получим $\gamma_{D_{1}}(t, x)=\int g(t, x, x) d x$, после этого применим определение (12) обобщенной дзета-функции с учетом (42).

\section{5. КВАНТОВАНИЕ РЕШЕНИЯ ТИПА КИНКА ДЛЯ МОДЕЛИ СГ}

Представим теперь в деталях формулы, имеющие место для кинков модели СГ. Подстановка выражений из (25), (29) приводит к появлению расходимости в интеграле (35) для $\widehat{\gamma}(p)$. Чтобы произвести регуляризацию, обратимся к выражению (31) с соответствующим модулем эллиптической функции $(k=1)$

$$
G(p, x)=G_{\mathrm{c}}(p)+G_{\mathrm{k}}(p, x)=\frac{1}{2 \sqrt{p+b^{2}}}+\frac{b^{2} \operatorname{sech}^{2}(b x)}{2 p \sqrt{p+b^{2}}} .
$$

Слагаемое $G_{c}(p)$ в этом выражении является диагональной частью функции Грина (решения уравнения (24)) для постоянного потенциала, а кинковая составляющая может быть легко вычислена с помощью формул (25), (29).

Из представления (43) следует, что квантовые поправки в массу кинка (антикинка) появляются в двух местах: во-первых, изменяется $\nu=m^{2}$ в выражении (11),

$$
\gamma_{D_{0}}(t)=4 \pi^{(1-d) / 2} \frac{\Gamma(s+(1-d) / 2)}{\Gamma(s)} m^{d-1-2 s},
$$

во-вторых, поправка возникает в результате применения общей схемы, в которой выражение для функции Грина $G_{\mathrm{k}}(p, x)$ из (43) подставляется в аналог формулы $(10)$, что дает

$$
\gamma_{\mathrm{k}}(p)=\int_{-\infty}^{\infty} G_{\mathrm{k}}(p, x) d x=\frac{b}{p \sqrt{p+b^{2}}} .
$$

Это табличная функция, но здесь хотелось бы остановиться на объяснении этого результата, предполагая дальнейшее развитие метода. Заметим, что разрез для функции $\sqrt{p+b^{2}}$ выбирается вдоль оси $\operatorname{Re} p$ от $-\infty$ до $-b^{2}$, причем сверху разреза выбирается значение $\sqrt{p+b^{2}}=i \sqrt{\left|p+b^{2}\right|}$, снизу разреза - значение $\sqrt{p+b^{2}}=$ $-i \sqrt{\left|p+b^{2}\right|}$. После деформирования контура интегрирования получающийся при $t>0$ интеграл

$$
\gamma_{\mathrm{k}}(t)=1-\frac{b}{\pi} \int_{0}^{\infty} \frac{e^{\left(\xi+b^{2}\right) t}}{\left(\xi+b^{2}\right) \sqrt{\xi}} d \xi
$$

можно выразить с помощью функции ошибок. После некоторой замены переменных получаем

$$
\gamma_{\mathrm{k}}(t)=\operatorname{erf}(b \sqrt{t})=\frac{2 b \sqrt{t}}{\sqrt{\pi}} \int_{0}^{1} e^{-b^{2} t \tau^{2}} d \tau
$$




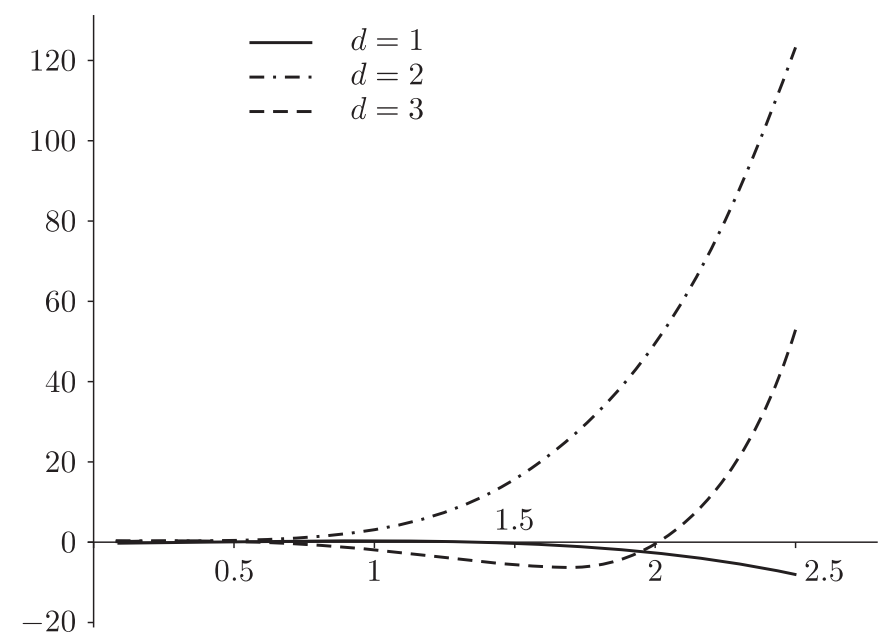

Зависимость поправочного члена для массы от параметра $m$ при $d=1,2,3$.

Преобразование Меллина от этого выражения приводит к регуляризованной дзетафункции для нашей задачи. Интеграл в выражении (12) сходится только при $\operatorname{Re} s<0$, но он допускает аналитическое продолжение на все $s \in \mathbb{C}$, кроме полюсов функции $\Gamma(s+1)$. Формула (12) с учетом (11) приводит при $b=m$ к выражению

$$
\zeta_{\mathrm{SG}}(s)=-4(4 \pi)^{d / 2} m^{d-1-2 s} \frac{\Gamma(s+1-d / 2)}{(2 s+1-d) \Gamma(s)} .
$$

Дифференцируя (48) по $s$,

$$
\begin{aligned}
\Delta S_{\mathrm{qu}}=\frac{\hbar}{2} & \frac{d\left(\zeta_{\mathrm{SG}}(s)\right)}{d s}=\frac{2 \hbar(4 \pi)^{d / 2} m^{d-2 s-1} \Gamma(s-d / 2+1)}{\Gamma(s)(2 s-d+1)^{2}} \times \\
& \times((d-2 s-1)(\Psi(-d / 2+s+1)-2 \ln m-\Psi(s))+2),
\end{aligned}
$$

в предельной точке $s=0$, получаем простое выражение для искомой поправки, которое можно легко представить графически. На рисунке представлена зависимость безразмерного поправочного члена $d\left(\zeta_{L}^{D}(0)\right) / d s$ от параметра $m$. Перейдем к заключению относительно выбора вида поправочного члена для массы поля Янга-Миллса. Интегралы в периодическом случае находились численно с помощью быстро сходящегося ряда для тета-функции. Читателю, интересующемуся более подробным описанием модели, видом различных функций, вычислениями и сравнениями, можно порекомендовать ознакомиться со статьей [7]. Некоторые современные работы открывают новые области применений метода [11]. Исследования динамики дислокационных особенностей с помощью модели Френкеля-Конторовой обеспечивают возможность прямой проверки эффектов квантовых солитонов, которые приводят к тому, что вклады кинка и эллиптических солитонов оказываются разными ввиду разной зависимости энергии от параметров. Интересно применить полученные здесь результаты в реальной термодинамике кристаллов в рамках подхода статистической 
физики [17] или с помощью прямого вычисления функции отклика с соответствующими измерениями [18]. Очень схожие с нашими результаты для кинков модели СГ (и некоторых других моделей) в $(1+1)$-случае опубликованы в работе [19].

Благодарности. Автор благодарит Г. Квятковского за ценные обсуждения и за помощь в построении графика зависимости массы кинка модели СГ от параметра $m$.

\section{Список литературы}

[1] O. M. Braun, Hu Bambi, A. Zeltser, Phys. Rev. E, 62:3 (2000), 4235-4245.

[2] S. V. Ketov, Fortschr. Phys., 45:3-4 (1997), 237-292; arXiv: hep-th/9611209.

[3] W. Nahm, Phys. Lett. B, 90:4 (1980), 413-414.

[4] E. Corrigan, P. R. Wainwright, S. M. J. Wilson, Comm. Math. Phys., 98:2 (1985), 259-272.

[5] J. A. Tuszyński, J. M. Dixon, A. M. Grundland, Fortschr. Phys., 42:4 (1994), 301-337.

[6] M. Pawellek, J. Phys. A, 42:4 (2009), 045404; arXiv: 0802.0710v1.

[7] S. Leble, Quantum corrections to static solutions of Nahm equation and Sin-Gordon models via generalized zeta-function, arXiv: 0806.2679.

[8] A. Comtet, A. Bandrauk, D. K. Campbell, Phys. Lett. B, 150:1-3 (1985), 159-162.

[9] R. F. Dashen, B. Hasslacher, A. Neveu, Phys. Rev. D, 10:12 (1974), 4114-4129; 11:12 (1975), 3424-3450; L. D. Faddeev, V. E. Korepin, Phys. Rep., 42:1 (1978), 1-87.

[10] Р. В. Коноплич, ТМФ, 73:3 (1987), 379-392; 78:3 (1989), 444-457.

[11] M. Bordag, A. S. Goldhaber, P. van Nieuwenhuizen, D. Vassilevich, Phys. Rev. D, 66:12 (2002), 125014; arXiv: hep-th/0203066.

[12] В. А. Фок, Изв. АН СССР. Сер. физ., 1937, № 4-5, 551-568.

[13] S. Leble, "Nahm equations as an integrable system: difference Lax pair, gauge-Darboux covariance, chains and solutions", Selected Problems of Moderm Mathematics, Proc. of 200-year of K. Jacobi birthday conf., Kaliningrad State Univ., Kaliningrad, 2005, 97-99.

[14] Г. Бейтмен, А. Эрдейи, Высшие трансцендентные функиии. Функиии Бесселя, функции параболического иилиндра, ортогональные многочлены, Справочная математическая библиотека, Наука, М., 1966.

[15] M. Adler, J. Moser, Comm. Math. Phys., 61:1 (1978), 1-30.

[16] E. D. Belokolos, A. I. Bobenko, V.Z. Enol'skii, A. R. Its, V. B. Matveev, Algebro-Geometric Approach to Nonlinear Integrable Equations, Springer, Berlin, 1994.

[17] A. Seeger, Mat. Sci. and Engrg. A, 370:1-2 (2004), 50-66.

[18] H. Koizumi, M. Tachibana, K. Kojima, I. Yonenaga, Phys. B, 401-402 (2007), 691-694.

[19] A. Alonso Izquierdo, W. García Fuertes, M. A. González León J. Mateos Guilarte, Nucl. Phys. B, 635:3 (2002), 525-557. 